\title{
EL INTERNET DE LAS COSAS Y SU APLICACIÓN EN LA PANDEMIA CONTRA EL
} COVID-19

AUTORES: Shayla Ayllin Cevallos Flores ${ }^{1}$

Lucas Arteaga Roddy Steven ${ }^{2}$

Raquel Azucena Pita Salazar ${ }^{3}$

Hilda Brigitte Quijije Quiroz ${ }^{4}$

Mario Javier Marcillo Merino ${ }^{5}$

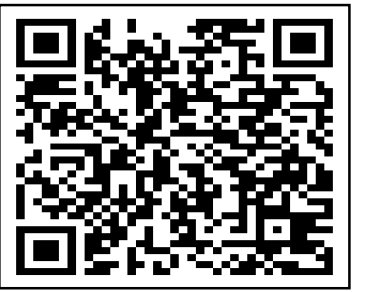

\section{DIRECCIÓN PARA CORRESPONDENCIA: (lucas-roddy9800@unesum.edu.ec)}

Fecha de recepción: 20/01/2021

Fecha de aceptación: 20/09/2021

\section{RESUMEN}

Esta investigación se llevó a cabo para presentar la existencia acerca de la IoT y los avances contemplados desde su implementación, desde la perspectiva enmarcada en su utilización en los diversos ámbitos tecnológicos, así como y a nivel material en el desarrollo de prototipos teniendo en cuenta el apogeo de los avances tecnológicos actuales para generar nuevos inventos. El objetivo primordial del internet de las cosas es mejorar los desafíos que podrían dificultar la realización de sus potenciales beneficios, uno de ellos se centró en la pandemia del covid-19 que afecto innumerables ámbitos sociales políticos y económicos, así como investigaciones de campo que en estos tiempos pudieron haberse lanzado al mercado facilitando las tareas en diversas áreas. Dentro del desarrollo de la investigación se abordaron los diferentes métodos científicos tales como métodos teóricos que implica histórico-lógico, análisis-síntesis y método bibliográfico. Los resultados demostraron el progreso de la tecnología y la calidad tecnológica por lo tanto se concluyó que el internet de las cosas es la herramienta del futuro que se conjuga con ramas de la mecatrónica y la inteligencia artificial para desbordar el boom de innovación.

PALABRAS CLAVE: Apogeo; investigaciones; tecnología.

\section{THE INTERNET OF THINGS AND ITS APPLICATION IN THE COVID-19 PANDEMIC}

\footnotetext{
${ }^{1}$ Cevallos Flores Shayla Ayllin (Ingeniera en Tecnología de la información. Universidad Estatal del Sur de Manabí. Jipijapa, Manabí y Ecuador. E-mail:cevallos-shayla8767@unesum.edu.ec)

${ }^{2}$ Lucas Arteaga Roddy Steven (Ingeniera en Tecnología de la información. Universidad Estatal del Sur de Manabí. Jipijapa, Manabí y Ecuador. E-mail:lucas-roddy9800@unesum.edu.ec)

${ }^{3}$ Pita Salazar Raquel Azucena (Ingeniera en Tecnología de la información. Universidad Estatal del Sur de Manabí. Jipijapa, Manabí y Ecuador. E-mail:pita-raquel0556@unesum.edu.ec)

${ }^{4}$ Quijije Quiroz Hilda Brigitte (Ingeniera en Tecnología de la información. Universidad Estatal del Sur de Manabí. Jipijapa, Manabí y Ecuador. E-mail:quijije-hilda2868@unesum.edu.ec)

${ }^{5}$ Ing. Marcillo Merino Mario Javier(Ingeniera sistemas computacionales. Docente - Universidad Estatal del Sur de Manabí. Jipijapa, Manabí y Ecuador. E-mail:mario.marcillo@unesum.edu.ec)
} 


\begin{abstract}
This research was carried out to present the existence about the IoT and the advances contemplated since its implementation, from the perspective framed in its use in the various technological fields, as well as and at the material level in the development of prototypes taking into account the apogee of current technological advances to generate new inventions. The primary objective of the internet of things is to improve the challenges that could hinder the realization of its potential benefits, one of them focused on the pandemic of covid-19 that affected countless social, political and economic areas, as well as field research that in these times could have been launched to the market facilitating tasks in various areas. Within the development of the research, the different scientific methods such as theoretical methods involving historical-logical, analysis-synthesis and bibliographic method were approached. The results demonstrated the progress of technology and technological quality therefore it was concluded that the internet of things is the tool of the future that combines with branches of mechatronics and artificial intelligence to overflow the innovation boom.
\end{abstract}

KEYWORDS: Apogee; research; technology.

\title{
INTRODUCCIÓN
}

El mundo avanza y presenta cambios constates y radicales, y hoy en día la tecnología está a la orden del día, uno de esos avances es la denominada IoT o Internet de las cosas, esta tecnología tiene la capacidad de transformar cualquier objetivo inerte en inteligencia, permitiendo darle una identidad propia, entre otros aspectos como la capacidad de comunicarse e interactuar con otros objetos y con los seres humanos.

La digitalización en constante trasformación organizada por estereotipos tecnológicos ha cambiado desde aspecto laborales, áreas de la medicina, medio ambiente entre otros, ha permitido la interacción entre el hombre y la máquina determinando puntos clave en donde la dependencia de IA, Robótica, mecatrónica y el internet de las cosas van de la mano, son requeridas y fabricadas a la necesidad de cada individuo. Como resultado, COVID-19 bien podría haber sido la consagración de Internet de las Cosas (IoT).

Recientemente (Ahmed, 2020) argumenta sobre el impacto de la COVID-19, se ha dejado sentir en países, comunidades y personas de muchas maneras, desde cierres de colegios hasta cifras de fallecidos alarmantes. Conforme los gobiernos se apresuraban en atajar estos problemas, fueron surgiendo diferentes soluciones basadas en diferentes tecnologías, incluidas las que conforman el llamado Internet de las Cosas (IoT, Internet of Things), para ayudar a poner coto a la crisis sanitaria mundial. Como resultado, de la pandemia puede haberse convertido en el catalizador definitivo para la adopción a gran escala de las tecnologías IoT.

En este aspecto el uso del IO de manera convencional es cada vez mucho más real y estaría al alcance de la mano en cuestión de años, no solo en instituciones privadas y públicas sino para un público en general, sus usos estarían dados en entornos empresariales, educativos, comerciales y caseros. Entonces, ¿Cómo puede ayudarnos IoT o en la próxima pandemia? ¿Deberíamos seguir apostando en la aplicación de la IoT en los diferentes entornos? A lo largo de este artículo exploraremos las diferentes áreas en las que IoT nos ha ayudado a superar la peor fase de la pandemia y cómo la COVID-19 ha cambiado nuestra percepción de IoT para siempre.

14 UNESUM-Ciencias. Publicación cuatrimestral. Vol. 5, Año 2021, No. 6 (Especial Ingenierías) 


\section{DESARROLLO}

\section{EI Internet De Las Cosas}

El Internet de las Cosas o IoT es la interconexión digital de los objetos cotidianos con Internet, convirtiéndose así en objetos inteligentes.El término fue acuñado en 1999 por Kevin Ashton, un investigador del MIT, mientras él y sus compañeros realizaban estudios en el campo de la identificación por radiofrecuencia y tecnologías de sensores.Es la tecnología capaz de convertir cualquier objeto inerte en inteligente, el famoso Smart, en inglés, dotándole de una identidad (virtual) propia, así como la capacidad de empatizar, comunicarse e interactuar con otros objetos y, por supuesto, con los humanos.(Leal, 2019).

El número de aplicaciones y servicios que pueden proporcionar es prácticamente ilimitado y se puede adaptar a muchos campos de la actividad humana, facilitando y mejorando su calidad de vida en múltiples formas.

\section{Inconvenientes del Internet de las Cosas destacan los siguientes:}

$\checkmark$ La información no se encuentra cifrada, por lo que no nos ofrece un total control de los dispositivos.

$\checkmark$ Requiere de una inversión en tecnología.

$\checkmark$ Dependencia de las organizaciones que ofrecen estos servicios.

$\checkmark$ Reducción de la intimidad.

$\checkmark$ Brechas tecnológicas entre ciudades y en una misma sociedad.

$\checkmark$ El software no es totalmente seguro y puede ser hackeado.

$\checkmark$ Falta de compatibilidad, ya que el IoT no está estandarizado.

$\checkmark$ Desconocimiento de qué información es fiable y, por tanto, valiosa.

$\checkmark$ Generación de residuos (Ricaurte, 2020).

\section{El intenet de las cosas (IoT) y el trabajo en remoto}

El Internet de las Cosas consta de cuatro componentes. Sensores, Redes, la nube y las aplicaciones (SNCA, Sensors, Networks, Cloud, and Applications), y el COVID-19 ha disparado su adopción por las medidas de teletrabajo que muchas empresas se han visto obligadas a adoptar. Antes de la pandemia, las tecnologías Iot que más interés habían despertado en el sector empresarial eran los sensores (84\%), las de procesamiento de datos (77\%) y las plataformas en la nube (76\%)

\section{Teletrabajo}

La pandemia del Coronavirus ha obligado a muchas de las empresas a optar por el teletrabajo. Si bien la idea del Teletrabajo surgió hace 50 años, durante la crisis del petróleo, como una forma de reducir el uso de recursos no renovables, ha sido en los últimos años, con el boom de las Tecnologías de la Información y la Comunicación, cuando realmente ha empezado a implantarse de una forma más horizontal en todos los sectores.

Uno de los grandes beneficios de este sistema es el ahorro de hasta $30 \%$ en infraestructura y aumento del nivel de satisfacción del trabajador, gracias a la mayor autonomía, flexibilidad, y facilidad para conciliar la vida laboral y la personal.

\section{Tipos De Teletrabajo}

\section{- Teletrabajo Domiciliario}


Es aquel que se realiza desde el domicilio del trabajador, la persona pasa toda la jornada laboral en su casa.

\section{- Teletrabajo Móvil}

Es el practicado por aquellos trabajadores que, por la naturaleza itinerante de su trabajo, llevan a cabo su jornada laboral fuera de la oficina, desde distintos sitios.

\section{- Teletrabajo En Telecentro}

En este caso, el trabajador realiza toda o parte de su jornada en centros de trabajo habilitados mediante recursos como equipos informáticos, fuera de las oficinas centrales. (Anónimo, 2020)

\section{Ventajas}

\section{Para los empleados:}

$\checkmark$ Permite mayor flexibilidad horaria y reduce el estrés.

$\checkmark$ Facilita la conciliación entre la vida familiar y profesional.

\section{Para las empresas:}

$\checkmark$ Reducción de costes al minimizar el espacio y la logística de la oficina.

$\checkmark$ Mayor productividad al reducirse las interacciones superfluas entre empleados.

\section{Para la sociedad:}

$\checkmark$ Reducción de la contaminación al haber menos desplazamientos en coche y transporte público.

\section{Desventajas}

\section{Para los empleados:}

$\checkmark$ El sedentarismo crece y pueden aumentar los problemas físicos, como el dolor de espalda.

$\checkmark$ Aumenta el riesgo de no desconectar y de trabajar más horas de las habituales.

\section{Para las empresas:}

$\checkmark$ La identificación del empleado con la compañía puede reducirse al aumentar el aislamiento.

$\checkmark$ El control del rendimiento de los empleados se vuelve más complejo desde la distancia.

\section{Para la sociedad:}

$\checkmark$ Reducción de las relaciones interpersonales que son la base de la sociedad.

$\checkmark$ Aumento de las diferencias sociales al existir una brecha en el acceso a las TIC. (Redondo, 2020)

\section{¿Es el Blockchain un arma contra la COVID-19?}

Ha surgido un nuevo recurso para el mercado laboral: el blockchain. Este ecosistema de cadena de bloques que funciona con una base de datos cifrada y segura, frecuentemente es relacionado con el mundo de las criptomonedas, pues es allí donde se efectúan sus transacciones, y otros servicios de índole financiero. Sin embargo, sus usos se extienden a otros ámbitos en los que se intercambian y alojan contratos, documentos, entre otros elementos, validados por terceros de manera independiente a través de unos nodos en los que la base de datos se actualiza y se deja registro del movimiento. (Aguirre, 2020)

Blockchain permite compartir cualquier transacción/información en tiempo real entre los diferentes nodos de una cadena, de manera segura e inmutable. En este caso, de haber existido una plataforma blockchain que conectara a la OMS, los Ministerios de Salud de cada país e incluso los principales hospitales de cada país, diseñada para compartir información en tiempo real sobre cualquier evento sanitario de manera inmediata, quizás el mundo hubiera podido reaccionar mucho antes frente a la pandemia. 
El uso de una plataforma blockchain para compartir información podría haberle ahorrado al planeta mucho dolor y muchas muertes. Se trata de un área de convergencia para el IoT y Blockchain, en la que blockchain se encargaría de garantizar la seguridad y trazabilidad de toda la información provista por los sensores/nodos a través de las redes disponibles para su procesamiento en la nube y presentación a través de aplicaciones. (Banafa, El Internet de las Cosas y COVID-19, 2020)

\section{IoT en el sector de la salud}

- Monitoreo de cuarentena. Controlar el cumplimiento de la cuarentena para aquellos infectados o sospechosos de haber contraído el coronavirus es muy importante para los gobiernos y las agencias de salud. Por lo tanto, muchos países están utilizando la tecnología IoT y GPS para rastrear el movimiento de las personas bajo observación. Hong Kong, por ejemplo, utilizó tecnología capaz de crear un perímetro virtual mediante GPS, RFID, Wi-Fi, Bluetooth y red celular.

- Desinfección y áreas hospitalarias. Se utilizan robots autónomos equipados con luz ultravioleta de alta intensidad para destruir el ADN del virus en instalaciones médicas en Italia, Estados Unidos y China. Los robots tienen conexión Wi-Fi y se pueden controlar a través de aplicaciones de forma remota.

- Registro privado de proximidad. Las empresas que desean volver a sus actividades laborales deben ofrecer soluciones para proteger a sus empleados. Una solución para mantener la distancia entre los empleados es una cerca virtual a nivel local. El dispositivo conectado a una red local activa una señal de alerta cuando dos o más personas están demasiado cerca la una de la otra. (Signals, 2020)

\section{IoT y el comercio electrónico}

El e-commerce, tanto a pequeña escala como dentro de los principales Marketplace, se presenta como una herramienta capaz de ayudar a las Pymes y Autónomos a minimizar las consecuencias de la crisis del coronavirus.

A la hora de vender de forma online, las pequeñas empresas y comercios de nicho pueden valorar diferentes opciones. Por un lado, la venta a través de un canal propio ofrece la ventaja de presentar los productos en el propio contexto de la marca, lo que da una visión holística de la misma y permite al usuario conocer toda su gama de productos o servicios. Asimismo, el vendedor evita los intermediarios en la venta y cuenta con la capacidad de decidir más libremente sobre la presentación del producto, de una forma personalizada y acorde con la imagen de marca. Por otro lado, la venta mediante un marketplace supone para los negocios un plus de visibilidad y posicionamiento, ya que éstos cuentan con una estrategia de SEO optimizada. Asimismo, también permite a los negocios acceder a un público mucho más amplio, al estar ubicado en una gran plataforma, y facilita la venta al extranjero, ya que elimina barreras previas técnicas o administrativas. Para optimizar sus ventas y alcanzar un público mayor, las empresas también pueden implementar un modelo mixto entre el canal propio y el Marketplace. (Center Hub, 2020).

\section{DISCUSIÓN Y RESULTADOS}

\section{Análisis Situacional}

Frente a las dificultades causada por la pandemia mundial de COVID-19, permitió que "el mercado de IoT supere el uso y el ingreso de las plataformas IoT en un aumento considerables de 
un 20\% en el primer trimestre del 2020 en comparación del 2019, logrando una disrupción económica más de 66.000 millones de dólares en 3 primero meses del 2020; lo que las convierte en una parte fundamental de la hoja de ruta tecnológica a corto plazo en muchas organizaciones. Ayudando a interconectar dispositivos, sensores para brindar data necesaria como ayuda a los empleadores a monitorear la salud y el bienestar de su personal” (lighting, 2020).

Según el último estudio Smart lightingde Juniper Research también ve "que la pandemia COVId19 impulsará la adopción de IoT en la industria de la salud. Señala que la tensión en los sistemas de salud causada por la crisis ha puesto de relieve los posibles beneficios de eficiencia que se pueden obtener del monitoreo remoto en la atención médica, sin embargo con la continuación la pandemia se impulsaron la adopción IoT en las vigilancias de detectar a las personas con temperaturas altas y atención a las distancias para reducir al mínimo las interacciones con el público en la aplicación de lugares públicos, aeropuertos, centro comerciales, terminales como primer filtro para la prevención y cuidado para la ciudadanía, siendo en si una gran tendencia y apoyo para organizaciones.”(lighting, 2020).

\section{ESTADÍSTICAS}

\section{IoT y la Telemedicina}

La pandemia de la COVID-19 adopto el IoT y en muchos sectores; según como nos indica Ahmed Banafa que "especialmente en la atención sanitaria, ahora que la crisis ha provocado en poner de relieve lo beneficioso para disponer de sistemas remotos de vigilancia y asistencia sanitaria A pesar de que se trata de un sector en el que la implantación de tecnologías IoT se ha producido muy lentamente o los recursos no son suficientes para continuar estos sistemas de ayudar remota, los institutos de salud de todo el mundo están teniendo muchos problemas para ofrecer atención sanitaria y reducir el riesgo de exposición”(Banafa, bbva open mind, 2020).

Por falencias que se da en países latinoamericanos y países no industrializados como la:

- Conectividad: falta de acceso a equipos y conexión a Internet.

- Habilidades: digitales por parte de los médicos o asistentes.

Tabla 1. América Latina: digitalización de la cadena de aprovisionamiento (2019)

\begin{tabular}{l|c|c|c}
\hline & $\begin{array}{c}\text { Porcentaje } \\
\text { de empresas } \\
\text { conectadas a } \\
\text { Internet }\end{array}$ & $\begin{array}{c}\text { Porcentaje } \\
\text { de empresas } \\
\text { que usan } \\
\text { banca } \\
\text { electrónica }\end{array}$ & $\begin{array}{c}\text { Porcentaje de } \\
\text { empresas que } \\
\text { usaan Internet } \\
\text { para adquisición } \\
\text { de insumos }\end{array}$ \\
\hline Argentina & $94,93 \%$ & $79,60 \%$ & $45,80 \%$ \\
\hline Brasil & $96,40 \%$ & $88,00 \%$ & $66,00 \%$ \\
\hline Chile & $86,16 \%$ & $84,37 \%$ & $28,80 \%$ \\
\hline Colombia & $92,81 \%$ & $95,39 \%$ & $37,00 \%$ \\
\hline Ecuador & $93,89 \%$ & $47,06 \%$ & $13,90 \%$ \\
\hline México & $94,61 \%$ & $76,60 \%$ & $13,47 \%$ \\
\hline Perú & $94,00 \%$ & $34,20 \%$ & $15,20 \%$ \\
\hline Uruguay & $93,39 \%$ & $68,35 \%$ & $38,43 \%$
\end{tabular}

Fuente1: análisis Telecom Advisory Services.

Según El banco interamericano de desarrollo, nos dice que "la pandemia del COVID-19 y las medidas de contención incluida están teniendo un impacto económico y social demoledor en el tejido empresarial de América Latina y el Caribe. especialmente en las mipymes (micro, pequeñas y medianas empresas), las cuales representan más del 99\% de las firmas en la región,

18 UNESUM-Ciencias. Publicación cuatrimestral. Vol. 5, Año 2021, No. 6 (Especial Ingenierías) 
más de un 22\% del PIB y un 61\% del empleo, de acuerdo con datos de la CEPAL (Pablo Angelelli, 2020)

Tabla 2. Índice de Desarrollo avance Digital (2019) Tabla 3 Red de colaboraciones internacionales

Fuente2: análisis Telecom Advisory Services.
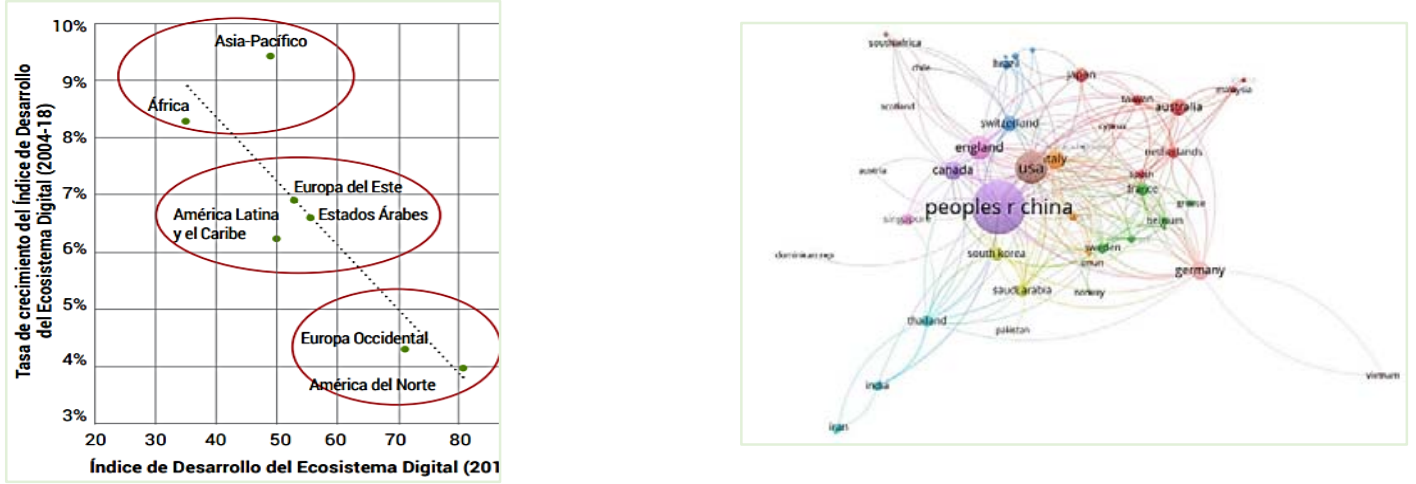

Fuente3 Hossain (2020)

Según Ahmed Banafa que los beneficios significativos para las tecnologías que "van en conjuntos al Internet de las Cosas Médicas (IoMT), junto con las tecnologías en la nube y la IA ofrecen una oportunidad para ayudar a los profesionales del sector sanitario a atender a sus pacientes, acceder a los datos y ofrecer tratamientos de manera no presencial, mediante el uso de dispositivos como termómetros inteligentes, dispositivos personales inteligentes, aplicaciones de rastreo y seguimiento y dispositivos médicos inteligentes" (Banafa, El Internet de las Cosas y COVID-19, 2020)

\section{CONCLUSIONES}

Podemos constatar que el IoT es el puente entre el mundo físico y el mundo digital, una conexión de cualquier dispositivo a Internet utilizando software y sensores integrados para comunicarse, recopilar e intercambiar datos entre sí, esta es una gran oportunidad para el mundo actual.

Los dispositivos conectados de forma inteligente permiten tener acceso a una mejor información para tomar mejores decisiones, aprovechar el IoT, tienen una ventana de posibilidades.

ElIoT es una tendencia creciente y duradera, transformadora que nos acercará y nos separará aún más, en respuestas al COVID-19 se destaca la importancia que tiene la tecnología digital en el fomento de sociedades inclusivas y justas.

\section{RECOMENDACIÓN}

Maximizar la eficiencia y uso del internet de las cosas, ya que es tiempo de expandir el paradigma del modelo "Digital".

Tomar decisiones que sean conscientes que el uso de las tecnologías de la información y comunicación en todos los ámbitos sociales no es una moda, sino una estrategia fundamental en la que se pueden encontrar soluciones tangibles. 
La tecnología se ha logrado adaptarse a las nuevas necesidades creadas por esta situación de pandemia global, para controlar la rápida expansión del COVID-19, pero nos gustaría recalcar que la tecnología no serviría de nada sin el esfuerzo diario de las personas que están luchando actualmente contra el virus.

\section{REFERENCIAS BIBLIOGRÁFICAS}

Aguirre, S. (28 de Agosto de 2020). El blockchain, la última arma para salir del desempleo y encontrar trabajo. Obtenido de yahoo! finanzas: https://es-us.finanzas.yahoo.com/noticias/blockchain-desempleo-encontrartrabajo170542209.html?guccounter=1\&guce_referrer=aHR0cHM6Ly93d3cuZ29vZ2xlLmNvbS8\&guce_referrer_si $\mathrm{g}=$ AQAAALcIYlVtJDNt6SeNscYQooHRFCdumzJsI2_NJJPFkWQelFUhNZKgcq613Z2RKnnFAQ2MmD00vMP-b

Ahmed, B. (2020). El Internet de las Cosas y COVID-19.

Anónimo. (05 de 2020). El teletrabajo: Una alternativa para las empresas en tiempos de Coronavirus. Obtenido de ticnegocios.camaravalencia.com: https://ticnegocios.camaravalencia.com/servicios/tendencias/el-teletrabajouna-alternativa-para-las-empresas-en-tiempos-decoronavirus/\#: :text=Con\%20la\%20pandemia\%20del\%20Coronavirus,abocadas\%20en\%20la\%20situaci\%C3 \%B3n\%20actual.

Banafa, A. (24 de agosto de 2020). bbva open mind. Obtenido de https://www.bbvaopenmind.com/tecnologia/mundo-digital/internet-de-las-cosas-y-covid-19/

Banafa, A. (24 de Agosto de 2020). El Internet de las Cosas y COVID-19. Obtenido de OpenMind BBVA: https://www.bbvaopenmind.com/tecnologia/mundo-digital/internet-de-las-cosas-y-covid-19/

Center Hub. (13 de Mayo de 2020). El ecommerce ha sido el «salvavidas» en tiempos de coronavirus. Obtenido de Contact Center Hub: https://contactcenterhub.es/el-ecommerce-ha-sido-el-salvavidas-en-tiempos-decoronavirus-2020-13-26843/

Leal, S. (15 de Septiembre de 2019). Desarollo Directivo. Obtenido de https://www.unir.net/empresa/desarrollodirectivo/transformacion-digital/que-es-el-iot-o-internet-de-las-cosas-y-como-impacta-en-tu-dia-a-dia/

lighting, s. (12 de mayo de 2020). smart lighting.Obtenido de https://smart-lighting.es/mercado-iot-crece-2020adopcion-iot-industria-sanitaria/

Pablo Angelelli, M. H. (25 de abril de 2020). Banco Interamericano de Desarrollo. Obtenido de publications iadb: https://publications.iadb.org/publications/spanish/document/Respuestas-al-COVID-19-desde-la-ciencia-lainnovacion-y-el-desarrollo-productivo.pdf

Redondo, T. (2020). El teletrabajo o cómo aunar conciliación familiar y productividad. Obtenido de IBERDROLA: https://www.iberdrola.com/talento/ventajas-y-desventajas-del-teletrabajo

Ricaurte, J. (17 de Septiembre de 2020). Direct. Obtenido de https://protegiendopersonas.es/las-principales-ventajasy-desventajas-del-internet-de-las-cosas/

Signals. (25 de Mayo de 2020). Internet de las Cosas y el sector salud en tiempos de pandemia. Obtenido de signalsiot: https://signalsiot.com/internet-de-las-cosas-y-el-sector-salud-en-tiempos-de-pandemia/

20 UNESUM-Ciencias. Publicación cuatrimestral. Vol. 5, Año 2021, No. 6 (Especial Ingenierías) 